\section{Immunophenotypic changes in leukemic blasts in children with relapsed/refractory B-cell precursor acute lymphoblastic leukemia after treatment with CD19-directed chimeric antigen receptor (CAR)- expressing $T$ cells}

Implementation of CD19 targeting with the bispecific engager blinatumomab and $\mathrm{T}$ cells harboring chimeric antigen receptor (CAR-T) has resulted in significant improvement in therapy results in children with relapsed/refractory (R/R) B-cell precursor acute lymphoblastic leukemia (BCP-ALL). ${ }^{1,2}$ However, some patients do not respond to therapy or experience relapse. ${ }^{1,3}$ Minimal residual disease (MRD) persistence or reappearance during CD19-directed therapy is a key sign predicting failure of pan-B-cell antigen targeting. Thus, accurate detection of residual tumor cells has emerged as a key tool in evaluating the efficacy of such immunotherapy. Loss of targeted antigen, which is the main escape mechanism for BCP-ALL under selective pressures of CD19-directed treatment, is a significant obstacle for MRD monitoring by multicolor flow cytometry (MFC). As MFC-MRD assessment is ordinarily based on CD19positive compartment analysis ${ }^{4}$ possible CD19 negativity of tumor cells increases the significance of other immunophenotypic markers for MFC data analysis. Nevertheless, antigens applicable for immunophenotypic aberrations evaluation are also known to undergo expression modulation during therapy. ${ }^{5}$ Recently, we demonstrated that each antigen useful for MFC-MRD detection can be either increased or decreased in children treated with blinatumomab. ${ }^{6}$ As longer CAR-T cell therapy persistence leads to prolonged pressure on leukemic cells compared to blinatumomab, ${ }^{3}$ this phenomenon could probably also affect the immunophenotypic stability of the leukemia, making MFC-MRD assessment in such patients very challenging. The current report briefly summarizes our data on MFC-MRD and relapse detection in patients treated with CAR-T cell therapy with a main focus on changes in the expression of markers that are relevant for MFC-MRD investigation.

We carried out a retrospective review of 39 pediatric patients with R/R BCP-ALL who received CAR-T cell therapy between February 2018 and September 2020 and in whom tumor blasts were detectable in the bone marrow (BM) at least once after CAR-T cell infusion. Among them, there were four patients resistant to CART cell therapy, 16 who experienced relapse $(>5 \%$ blast cells by MFC) and 19 children with blasts in the BM on MFC-MRD level. The characteristics of the patients, including cytogenetic data, are presented in the Online Supplementary Table S1. The study was approved by the Ethics Committee of the Dmitry Rogachev National Medical Research Center of Pediatric Hematology, Oncology and Immunology, and informed consent for the collection and investigation of samples and for participation in current study was obtained from patients' parents or legal guardians.

Subjects reported here were either included in a prospective trial of CD19 CAR-T cell therapy with the 4$1 \mathrm{BB}$ costimulatory domain (clinicaltrials gov. Identifier: NCT03467256) or treated on a compassionate use basis. Morphological examination and MFC-MRD detection in BM aspirates were performed before CAR-T cell infusion, on days 14 and 28 , and 2, 3, 4, 6, 8, 10,12, and 24 months after the beginning of CAR-T cell therapy. The immunophenotypic stability of leukemic cells was eval- uated only after one CAR-T cell therapy course.

All patients underwent routine diagnostic immunophenotyping and MRD detection by 8-10-color flow cytometry according to the standard protocols of the Moscow-Berlin group.,8 During the study period, MFC was performed on FACS Canto II, FACS Celesta (both from Becton Dickinson, BD, US), CytoFlex and Navios (both from Beckman Coulter, BC, US) flow cytometers. EuroFlow guidelines for machine performance monitoring were used. ${ }^{9}$ Cytometer Setup and Tracking beads (BD), Flow-Check Pro Fluorospheres $(\mathrm{BC})$ and CytoFLEX Daily OC Fluorospheres (BC) were used for daily cytometer optimization. Normal lymphocytes were used as the control for positivity/negativity definition. The tumor cell immunophenotype was analyzed with a focus on markers applicable for MFC-MRD investigation. The Online Supplementary Table S2 provides a list of monoclonal antibodies used for MFC-MRD monitoring. CD22 and CD24 were additionally studied as suggested by $\mathrm{S}$. Cherian et al. ${ }^{10}$ MFC-MRD results were controlled with IG/TR gene rearrangements and fusion genes monitoring by molecular techniques with high concordance obtained ( $85 \%$ of qualitatively concordant $\mathrm{MRD}$ results for $\mathrm{CD} 19$-negative relapses and 79\% for those who retained CD19 positivity). Expression of surface antigens was deemed positive if the antigen was expressed on more than $20 \%$ of tumor cells. ${ }^{7}$ An increase/decrease in the expression of each single antigen was defined as a change in the percentage of positive cells of more than $25 \%$. Proportions of patients with stable and changed expression of each single antigen between CD19-negative and CD19-positive entities were compared using Fisher's exact test.

The CD19 expression status on leukemic cells after CAR-T cell therapy is depicted in Figure 1. The blasts of all patients except one were totally CD19-positive prior to CAR-T administration. In the remaining patient, only $30 \%$ of BM cells expressed the target antigen, while in cerebrospinal fluid (CSF), all leukemic BCP were CD19positive. In five patients, the leukemic cells at relapse were CD19-positive, and in 11 relapsed patients, CD19 negativity was found. Two of 11 CD19-negative relapses displayed a small CD19-positive subpopulation (5\% and $15 \%$ respectively). Patients who were resistant to CAR$\mathrm{T}$ cell therapy had preserved CD19 expression. The blasts of children who had leukemic cells in the BM on MFC-MRD level only ( $\mathrm{n}=19)$ were either CD19-positive $(n=10)$ or CD19 negative $(n=9)$. CD19-negative leukemia appeared a little less frequent in children who have already received CD19 targeting (8 of $16,50 \%$ ) comparing to those who were not pretreated with blinatumomab (12 of $19,63 \%, P=0.506)$.

The immunophenotypic changes of leukemic cells in the 16 relapsed patients are presented in Figure 2A. For all antigens applicable for MFC-MRD assessment except CD58, expression changes were demonstrated, either increased or decreased expression (Figure 3). On the other hand, the proportion of patients with noted changes was relatively small: CD34 was found to be the most unstable antigen (changes in 4 of 16 patients, $25 \%)$. We did not find differences in the frequencies of changes in marker expression among CD19-negative and CD19-positive relapses (Figure 2A). The expression of $\mathrm{CD} 22$ and $\mathrm{CD} 24$, which are suggested to be candidates for CD19 substitution, ${ }^{10}$ displayed remarkable stability, although $\mathrm{CD} 24$ was tested only in part of the study group. We analyzed 19 patients who did not relapse but had leukemic cells at the MRD level in the BM by MFC at least once during the follow-up period. In 


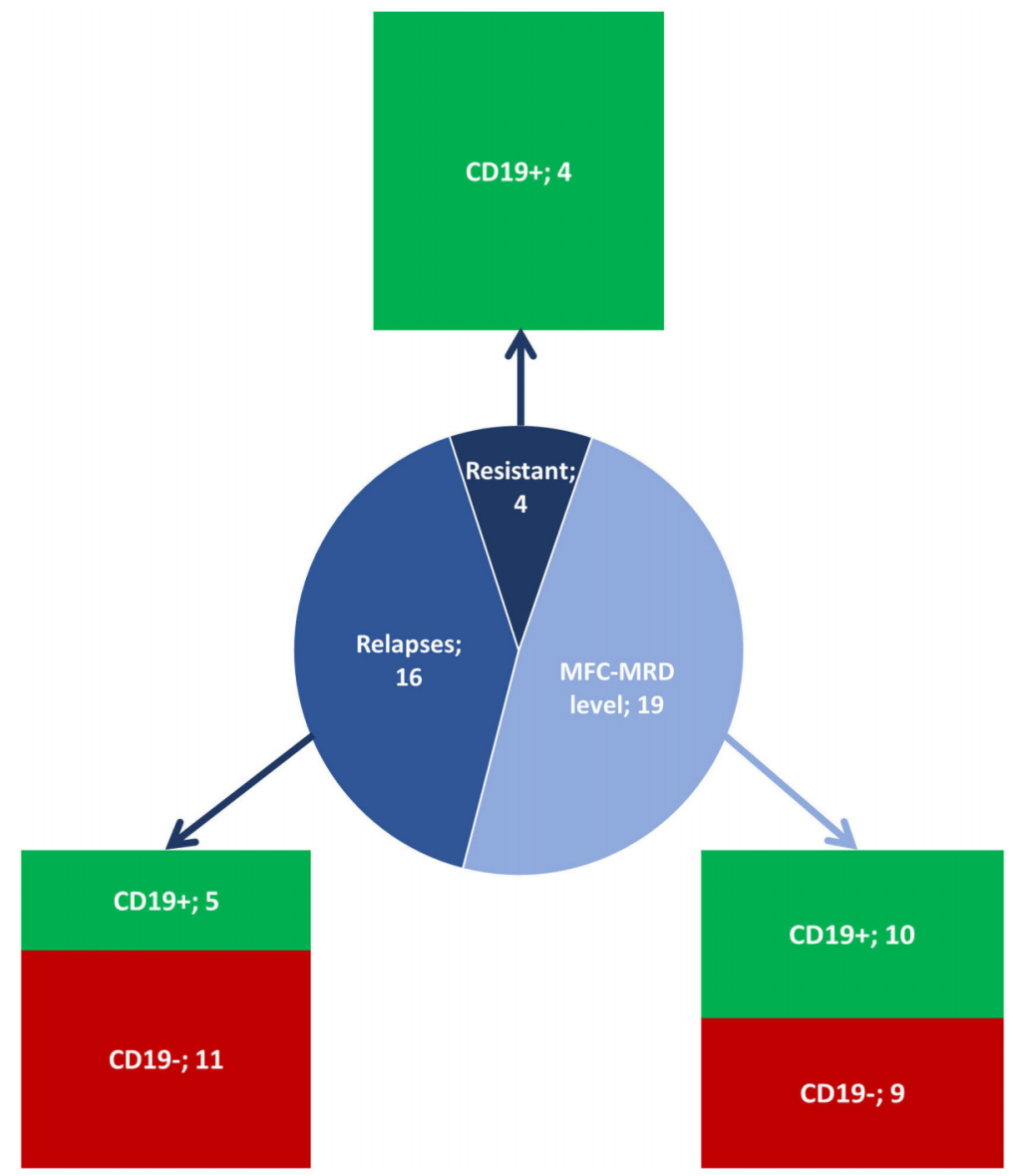

Figure 1. CD19 expression in the studied patients $(n=39)$. The study group included resistant patients $(n=4)$, patients with relapse (5 CD19-positive and 11 CD19-negative) and patients with blasts detected by multicolor flow cytometry (MFC) at the minimal residual disease (MRD) level in the bone marrow at least once $(n=19)$.

addition to explicable downmodulation of CD19 in nearly half of the patients (9 of 19), as in the relapsed patients, CD34 displayed the highest instability (expression changes in 10 of 19 patients), while other antigens again were rather stable, with no changes noted in the expression of CD38, CD58 and CD22 (Figure 2B). The leukemic cells in four resistant patients had a rather stable immunophenotypic profile with very rare cases of antigen expression changes (Figure 2C).

As CD19 is the best known target for immunotherapy in BCP-ALL, ${ }^{1,2}$ CD19-directed CAR-T cells are widely used as the salvage approach for $\mathrm{R} / \mathrm{R}$ patients. ${ }^{3}$ Thus, lack or preservation of CD19 expression is crucial for further treatment strategy choice. D. Libert et al. have shown that CD19 negativity occurs in nearly $65 \%$ of relapses after CAR-T cell application ${ }^{11}$ with a massive disproportion in frequency between 4-1BB- and CD28containing platforms $(85 \%$ vs. $22 \%$ of relapsed patients, respectively).$^{11}$ In addition, as discussed previously, ${ }^{6}$ loss of CD19 could break the well-established conventional algorithm of MFC-MRD gating, as cytometric residual leukemia detection is based on B-cell compartment investigation. ${ }^{4}$ In our study, 4-1BB CAR-T cells were used, and the frequency of CD19-negative relapses was $68.8 \%$. Taking these patients together with the resistant patients (all remained CD19-positive) and the patients with only MFC-detected MRD tumor cells, the total incidence of CD19 negativity was $51.3 \%$ in all children in whom tumor cells were detectable in the BM during follow-up after the first CAR-T cell therapy course. In all three patients in whom CD19-positive relapse was preceded by MFC-MRD reappearance, the residual leukemia had preserved expression of CD19 (Online
Supplementary Figure S1). Among the 11 CD19-negative relapses, six were preceded by CD19-negative MFC$M R D$, and one was preceded by a progressive decrease in CD19 expression on MFC-MRD, while in others, relapse developed directly after MFC-MRD negativity (Online Supplementary Figure S1).

As CD19 downregulation was noted in more than half of the patients, for antigens useful for primary B-lineage gating (CD10, CD22, CD24) ${ }^{10,12}$ not only the stability but also homogeneous positivity on tumor cells is vital. In the current study, neither of these antigens displayed significant changes, although their expression prior to CAR-T cell infusion was not always satisfactory. Indeed, in six of 33 and eight of 25 patients, less than $90 \%$ of the leukemic population before the CAR-T cell course was CD22-positive (median 64\%, range, $4-73 \%$ ) and CD24positive (median $0 \%$, range, $0-79 \%$ ), respectively. As expected, ${ }^{13}$ in four patients with low CD22 and in five patients with low CD24, rearrangements involving the KMT2A gene (KMT2A-r) were found. CD10 negativity/low expression (median 9\%, range, $0-52 \%$ ) was also not restricted to KMT2A-r cases: it was found in 13 patients ( 7 with $K M T 2 A-r)$ and remained stable in nine children. Moreover, in two of three cases of CD10 upmodulation, this marker expression after CAR-T cell therapy was still not total.

Previously, we showed the results of a similar study that included patients with R/R BCP-ALL treated with blinatumomab only. ${ }^{6}$ Compared to that after blinatumomab treatment, the proportion of cells with CD19 loss after CAR-T cell treatment was significantly higher (51.3\% vs. $27.1 \%)$, although no lineage switches were noted in the current work (comparing 3 of 30 relapses 


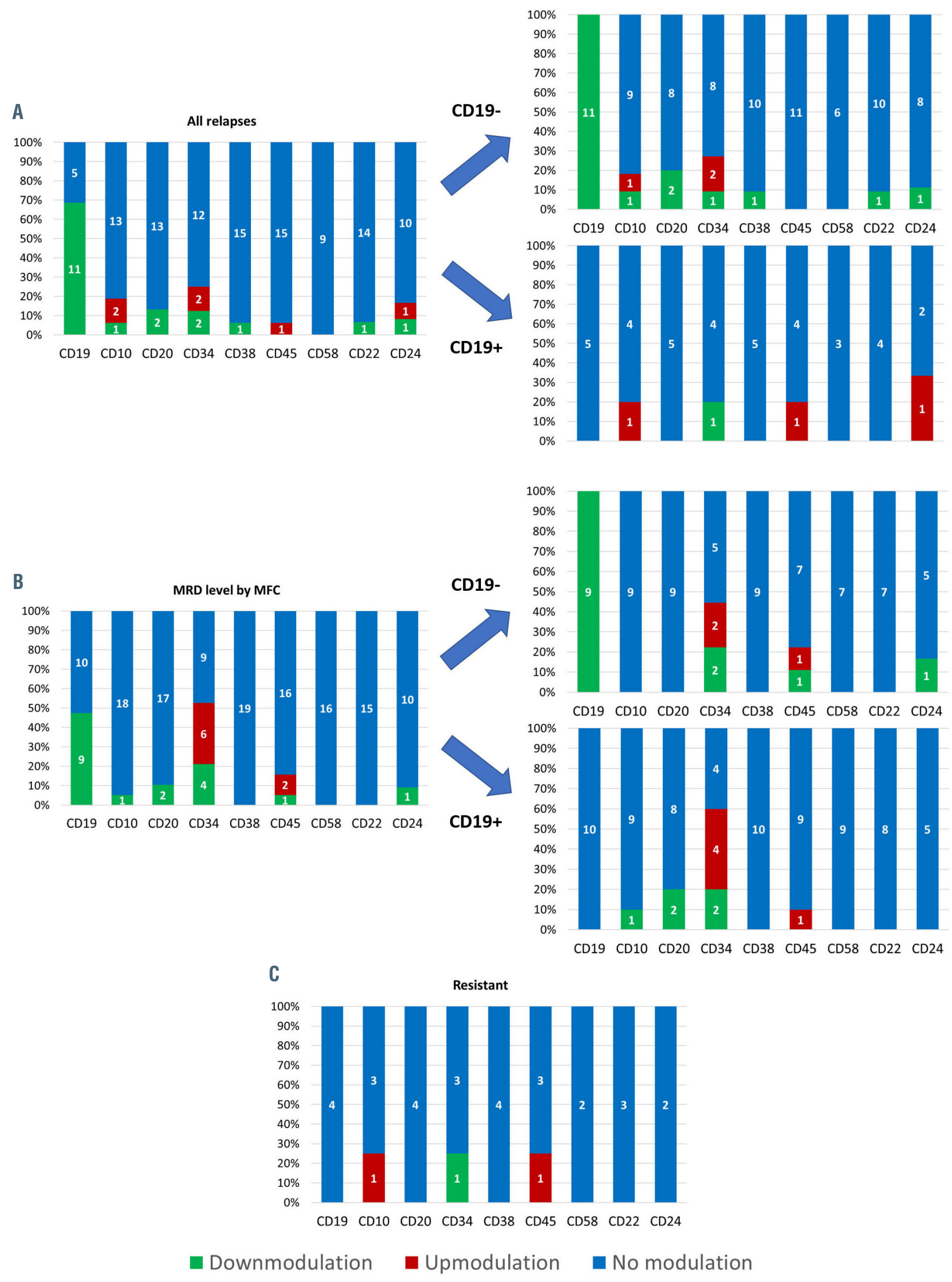

Figure 2. Frequency of changes in the immunophenotype of leukemic blasts. (A) Relapsed patients. (B) Patients who had detectable blasts in the bone marrow only at the minimal residual disease (MRD) level. (C) Resistant patients. 
after blinatumomab). Generally, the antigen profile of leukemic cells after CAR-T treatment displayed relatively higher stability, although CD58 was the most stable and CD34 was the most unstable marker irrespective of the type of immunotherapy given. Moreover, no differences in the immunophenotype of CD19-negative and CD19-positive relapses and MFC-MRD persistence were noted after CAR-T cell therapy, while after BiTE treatment, CD45 and CD38 changed more frequently in the case of CD19 loss. ${ }^{6}$

With its peculiar immunophenotype and tendency towards lineage switch, KMT2A-r BCP-ALL cases require special solutions for MFC-MRD monitoring after CD19 targeting. In our cohort, there were seven patients with various types of KMT2A-r. Before CAR-T cell therapy, they were either CD10-negative $(n=6)$ or borderline CD10-positive $(n=1, C D 10$ decreased at MFC-MRD reappearance). Among six children with available CD22 and CD24 expression, only two were totally CD22-positive and one was CD24-positive. Moreover, in three patients of this subgroup, ALL recurrence was CD19. negative. Fortunately, no lineage switches were regis-

A
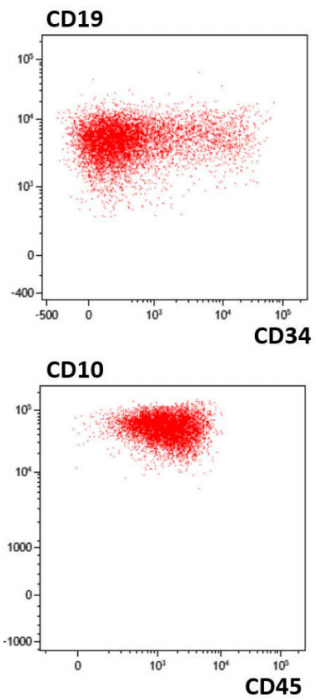

C
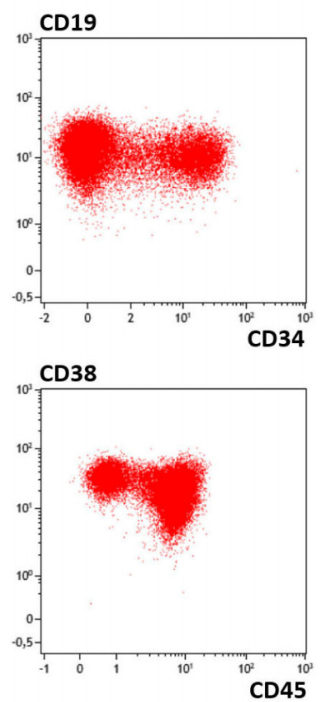

After CAR-T
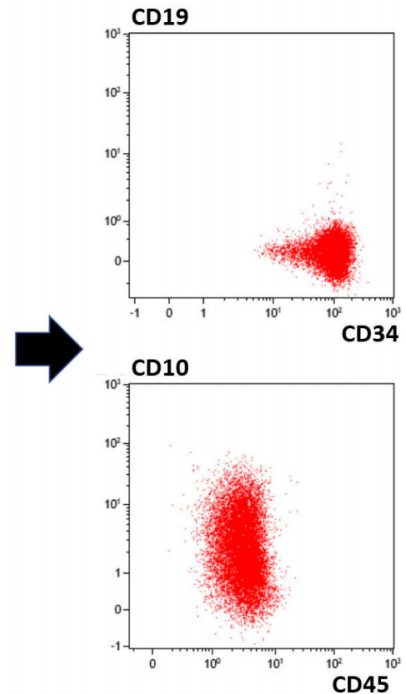

After CAR-T

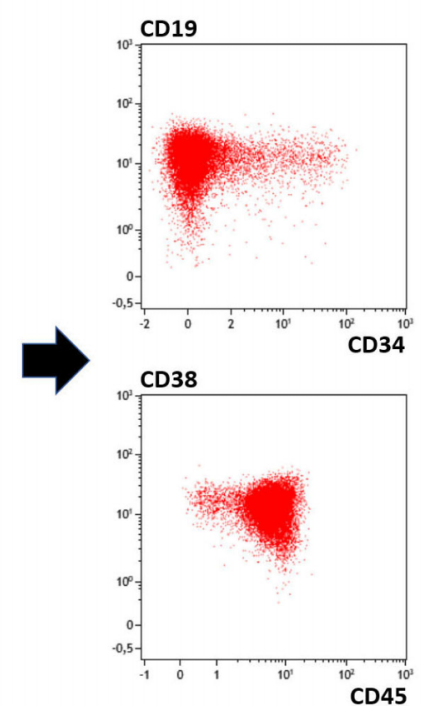

tered in this study (probably due to relatively few patients investigated), although they were rather frequent in case of blinatumomab application. ${ }^{6}$ Described immunophenotypic features make this genetic subgroup a very hard target for MFC-MRD technique. Being notably rare in the general BCP-ALL population, $K M T 2 A-r$ are present in a significant part of $\mathrm{R} / \mathrm{R}$ patients $^{14,15}$ (17.9\% in the current study). Considering this, addition of at least one early B-lineage antigen (iCD79a preferably) and careful analysis of the whole immunophenotype with searching both for initial antigenic patterns and for cells different from normal counterparts (with respect to specific regeneration patterns) ${ }^{16}$ could lead to reliable results, highly comparable with molecular techniques data $(86 \%$ with IG/TR rearrangements monitoring by next-generation sequencing and $98 \%$ with polymerase chain reaction-based fusion genes transcript detection). Moreover, additional MRD confirmation tools, such as fluorescence in situ hybridisation or chimerism studies ${ }^{17}$ from sorted suspicious cells, can help in MFC-MRD monitoring of such a complicated BCP-ALL subgroup.

B
Before CAR-T
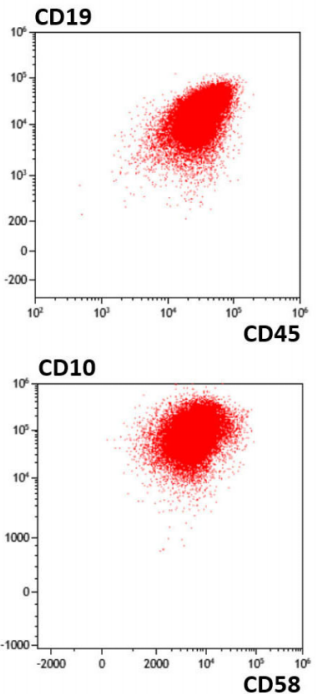

After CAR-T
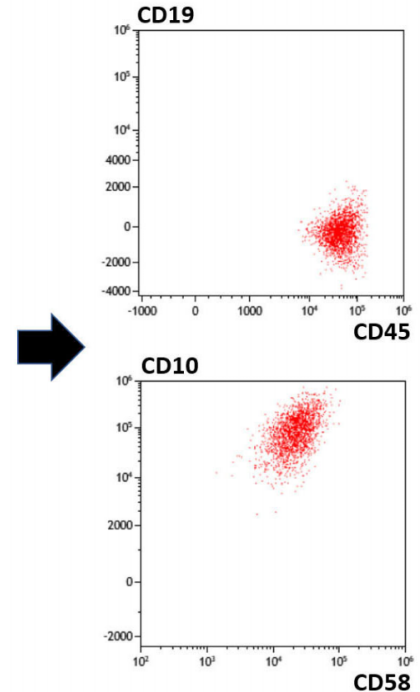

Figure 3. Representative examples of immunophenotypic plasticity in patients after $\mathrm{T}$ cells harboring chimeric antigen receptor. (A) Case of CD19-negative relapse. (B) Case of reappearance of CD19-negative minimal residual disease (MRD). (C) Case of phenotypically stable resistance to the immunotherapy. CAR-T: CD19-directed chimeric antigen receptor (CAR)expressing T cells. 
Despite the more stable antigen profile of leukemic cells after CAR-T cell therapy than after blinatumomab treatment, more frequent loss of CD19, which is the cornerstone of $\mathrm{B}$-lineage gating during conventional MFC$\mathrm{MRD}$ detection, necessitates usage of additional B-lineage antigens such as CD22, CD24, CD10 and iCD79a for primary B-cell compartment gating. Therefore, the final search for MFC-MRD becomes more sophisticat$\mathrm{ed}^{6,18}$ than conventional CD19-based methods, especially taking into account specific BM regeneration patterns that are more visible after CD19 targeting. ${ }^{16}$

Thus, considering the frequent loss of CD19 as well as the modulation of the expression of all other antigens relevant to MFC-MRD monitoring, a large panel of antibodies including additional B-lineage markers (CD22, CD24, iCD79a, etc.) combined with modified gating strategy and consideration of specific background variations, should be applied to increase the effectiveness of MFC-MRD detection in BCP-ALL patients after CD19 targeting by CAR-T cell therapy.

Ekaterina Mikhailova, Olga Illarionova, Larisa Shelikhova, Elena Zerkalenkova, Olga Molostova, Yulia Olshanskaya, Galina Novichkova, Alexey Maschan, Michael Maschan and Alexander Popov

Dmitry Rogachev National Medical Research Center of Pediatric Hematology, Oncology and Immunology, Moscow, Russian Federation

Correspondence:

ALEXANDER M POPOV - uralcytometry@gmail.com

doi:10.3324/haematol.2021.279677

Received: July 21, 2021

Accepted: December 2, 2021

Pre-published: December 16, 2021.

Disclosures: no conflicts of interest to disclose.

Contributions: EM and AP designed the study, acquired and analyzed cytometric data and wrote the paper; OI acquired and analyzed cytometric data; LSh collected clinical data and wrote the paper; EZ acquired and analyzed cytogenetic and molecular genetic data, and wrote the paper; OM collected clinical data; $\mathrm{YuO}$ acquired and analyzed cytogenetic and molecular genetic data; GN and MM designed the study and acted as the general supervisor; AM designed the study, acted as the general supervisor and wrote the paper.

Funding: the KMT2A rearrangement assessment study was supported by RFBR grant number 7-29-06052 and Presidential grant number MK-1645.2020.7 and 075-15-2020-338. Flow cytometric minimal residual disease evaluation was supported by RFBR grant number 18-29-09132.

\section{References}

1. Topp MS, Gokbuget N, Zugmaier G, et al. Phase II trial of the antiCD19 bispecific T cell-engager blinatumomab shows hematologic and molecular remissions in patients with relapsed or refractory Bprecursor acute lymphoblastic leukemia. J Clin Oncol. 2014;32(36):4134-4140.

2. Maude SL, Frey N, Shaw PA, et al. Chimeric antigen receptor T cells for sustained remissions in leukemia. $N$ Engl J Med. 2014;371(16):1507-1517.

3. Maude SL, Laetsch TW, Buechner J, et al. Tisagenlecleucel in children and young adults with B-cell lymphoblastic leukemia. N Engl J Med. 2018;378(5):439-448.

4. Dworzak MN, Gaipa G, Ratei R, et al. Standardization of flow cytometric minimal residual disease evaluation in acute lymphoblastic leukemia: multicentric assessment is feasible. Cytometry B Clin Cytom. 2008;74(6):331-340

5. Dworzak MN, Gaipa G, Schumich A, et al. Modulation of antigen expression in B-cell precursor acute lymphoblastic leukemia during induction therapy is partly transient: evidence for a drug-induced regulatory phenomenon. Results of the AIEOP-BFM-ALL-FLOWMRD-Study Group. Cytometry B Clin Cytom. 2010;78(3):147-153.

6. Mikhailova E, Gluhanyuk E, Illarionova $\mathrm{O}$, et al. Immunophenotypic changes of leukemic blasts in children with relapsed/refractory B-cell precursor acute lymphoblastic leukemia, who have been treated with Blinatumomab. Haematologica. 2020;106(7):2009-2012

7. Novikova I, Verzhbitskaya T, Movchan L, et al. Russian-Belarusian multicenter group standard guidelines for childhood acute lymphoblastic leukemia flow cytometric diagnostics. Oncohematology. 2018;13(1):73-82.

8. Popov A, Belevtsev M, Boyakova E, et al. Standardization of flow cytometric minimal residual disease monitoring in children with Bcell precursor acute lymphoblastic leukemia. Russia-Belarus multicenter group experience. Oncohematology. 2016;11(4):64-73.

9. Kalina T, Flores-Montero J, Lecrevisse Q, et al. Quality assessment program for EuroFlow protocols: summary results of four-year (2010-2013) quality assurance rounds. Cytometry A. 2015;87(2): 145-156.

10. Cherian S, Miller V, McCullouch V, et al. A novel flow cytometric assay for detection of residual disease in patients with B-lymphoblastic leukemia/lymphoma post anti-CD19 therapy. Cytometry B Clin Cytom. 2018;94(1):112-120.

11. Libert D, Yuan CM, Masih KE, et al. Serial evaluation of CD19 surface expression in pediatric B-cell malignancies following CD19-targeted therapy. Leukemia. 2020;34(11):3064-3069.

12. Mejstrikova E, Hrusak O, Borowitz MJ, et al. CD19-negative relapse of pediatric B-cell precursor acute lymphoblastic leukemia following blinatumomab treatment. Blood Cancer J. 2017;7(12):659.

13. De Zen L, Bicciato S, te Kronnie G, Basso G. Computational analysis of flow-cytometry antigen expression profiles in childhood acute lymphoblastic leukemia: an MLL/AF4 identification. Leukemia. 2003;17(8):1557-1565

14. Queudeville M, Schlegel P, Heinz AT, et al. Blinatumomab in pediatric patients with relapsed/refractory B-cell precursor acute lymphoblastic leukemia. Eur J Haematol. 2021;106(4):473-483.

15. Winters AC, Bernt KM. MLL-rearranged leukemias-an update on science and clinical approaches. Front Pediatr. 2017;5:4.

16. Mikhailova E, Semchenkova A, Illarionova O, et al. Relative expansion of CD19-negative very-early normal B-cell precursors in children with acute lymphoblastic leukaemia after CD19 targeting by blinatumomab and CAR-T cell therapy: implications for flow cytometric detection of minimal residual disease. Br J Haematol. 2021;193(3):602-612.

17. Semchenkova A, Brilliantova V, Shelikhova L, et al. Chimerism evaluation in measurable residual disease-suspected cells isolated by flow cell sorting as a reliable tool for measurable residual disease verification in acute leukemia patients after allogeneic hematopoietic stem cell transplantation. Cytometry B Clin Cytom. 2020;100(5):568-573.

18. Cherian S, Stetler-Stevenson M. Flow cytometric monitoring for residual disease in $B$ lymphoblastic leukemia post $\mathrm{T}$ cell engaging targeted therapies. Curr Protoc Cytom. 2018;86(1):e44. 